\title{
Cooperative Rotor Position Estimation of Active Magnetic Bearings in Flywheel energy storage system with Unsaturated Magnetic Bias
}

\author{
M. TANG, L.J. Ding, M. Li, H. Zhang, W. Wei F. Tang \& G. Chen \\ Electric power science research institute of state grid Sichuan electric power company, Chengdu, \\ 610071
}

KEYWORD: Self-sensing; active magnetic bearings; unsaturated magnetic bias; cooperative position ABSTRACT: A strategy of cooperative position estimation is proposed based on the differential control mode of active magnetic bearing (AMB). Given unsaturated magnetic bias, one of the opposing pole pairs must be unsaturated. The unsaturated poles could be tracked by the control current polarity. The position estimation from the unsaturated poles is always chosen as system feedback. Basic theory of unsaturated magnetic bias is discussed and the experimental method of measuring the maximum value of unsaturated current bias is presented. This cooperative position estimation is tested in the 4-DOF AMBs system. Static and dynamic suspension experiments are held to prove the feasibility of this method. Experimental results show that, the self-sensing AMBs system using this strategy exhibits stronger rejection to magnetic saturation. And this strategy proves the performance of force disturbance and increases system stability.

\section{Introduction}

Active magnetic bearing (AMB) is being employed for a variety of industrial rotating machineries for their unique features over conventional bearings. Their contact-free suspension provides no lubrication, no mechanical friction and so they potentially have the ability to achieve higher speed. With the development of AMB, high-reliability and low-cost become the main direction to pursue.

Self-sensing AMB offers an opportunity to achieve high-reliability and low-cost by replacing position sensing device with a specific form of signal processing which extracts rotor position information from coil current and voltage signals. The self-sensing AMB has been carried out for years by lots of researchers and many methods have been proposed in literature.

Generally, there are two broad categories of self-sensing AMB: state observation and modulation. The state observation approach was first proposed by Visher (1988). His work showed that the AMBs state model is controllable and observable with coil current and voltage measured. Rotor position is treated as one of the state variables which can be reconstructed by a Luenberger observer. But such a plant is very sensitive to variations of model parameter. The modulation approach is based on inductance measurement principle. Similar to inductance sensor, a high frequency signal with tiny amplitude is injected to the AMB's coils to measure the variation of coil inductance caused by the rotor displacement . Because of its high frequency (much higher than control signal frequency), rotor position can be demodulated by using a high-pass filter (HPF) and other signal process circuits. System robustness is then improved compared to the state observation approach.

For its high efficiency, the pulse width modulation (PWM) switching power amplifiers have been widely used in AMB. They act not only as actuators but also an excellent alternative for the high frequency source. In this case, the actuator itself is a position sensor, so there is no noncollocation problem which may introduce difficulties in stabilizing the system. More recent work focused on how to make good use of this high frequency source. However, it remains a challenge to make good use of this excellent alternative because of its intrinsic particularity.

The nature mechanism of position estimation is to measure the equivalent inductance of the coil. However, all this estimation is carried out by the premise of unsaturation for the reason that the equivalent inductance of coils would decrease rapidly once the ferromagnetic material reach saturation area. This paper proposed a strategy to solve this problem.

Differential control is widely used in AMB system, so the opposite pole pair must be in different degree of saturation. This paper tries to find a proper bias current that will keep an unsaturated pole at any condition. Cooperative rotor position estimation strategy is proposed and its effectiveness is verified by lots of experiments. 


\section{Inductor model of AMB and position estimation method}

Figure 1shows the 8-pole AMB rotor. For electrical circuit point of view, the coil of AMB equals to inductance load. Research on the relationship of coil equivalent inductance and air gap is the basic of self-sensing control.
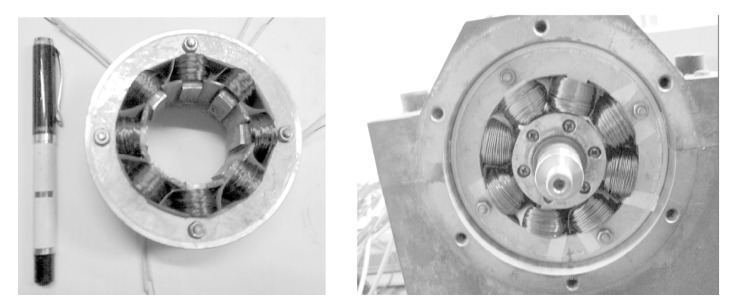

Figure 18 -pole stator of magnetic bearing

Vertical direction coil is selected for analysis as shown in Figure 2. $V$ is coil voltage, $I$ is coil current, $x$ is air gap, $l_{\mathrm{c}}$ is average length of magnetic loop in rotor and stator, $N$ is coli turns.

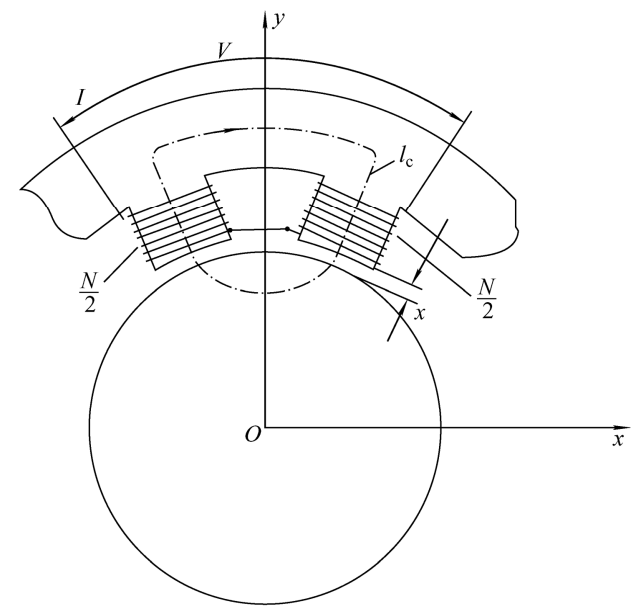

Figure 2 Demonstration of electromagnetic loop of stator and rotor

Coil voltage equation for AMB:

$V=N \frac{\mathrm{d} \Phi}{\mathrm{d} t}+R I$

Where $\Phi$-Main flux under the condition that the leakage flux is ignored.

$R$-Coil resistance.

The main flux can be expressed as:

$\Phi=\frac{N I}{R_{\mathrm{m}}}$

Where $R_{\mathrm{m}}$-Total reluctance of magnetic loop can be calculated as:

$R_{\mathrm{m}}=\frac{2 x+l_{\mathrm{c}} / \mu_{\mathrm{r}}}{\mu_{0} A}$

Where $\mu_{0}$-magnetic permeability of air.

$\mu_{\mathrm{r}}$-relative permeability of ferromagnetic material.

The total flux in the coil is:

$\Psi=L I=N \Phi$

From Equation 2-4, the coil equivalent inductance $L$ is: 


$$
L=\frac{\mu_{0} N^{2} A}{2 x+l_{\mathrm{c}} / \mu_{\mathrm{r}}}
$$

Where $l_{\mathrm{c}} / \mu_{\mathrm{r}}$ can be seen as an equivalent air gap for the magnetic circuit in rotor and stator. When the internal magnetic flied in the ferromagnetic material is not saturated, $\mu_{\mathrm{r}}$ is normally large, compared to air gap $2 x$, equivalent air gap $l_{\mathrm{c}} / \mu_{\mathrm{r}}$ is small enough to be ignored. So, the relationship between coil equivalent inductance and air gap is approximately inverse proportion. This is just the theoretical foundation of position estimation based on inductance measurement.

Commonly, inductance measurement method always uses a high frequency signal with a small magnitude injecting into the coil. The impedance of coil can be calculated from coil voltage and current response to specific frequency injected. However, in AMB systems driven by switching power amplifier, the PWM signal itself is a high frequency injection. For the current mode switching power amplifiers, the coil voltage in time domain is shown as follows:

$$
V(t)= \begin{cases}V_{\mathrm{s}} & k T_{\mathrm{s}}<t \leqslant k T_{\mathrm{s}}+\alpha_{k} T_{\mathrm{s}} \\ -V_{\mathrm{s}} & k T_{\mathrm{s}}+\alpha_{k} T_{\mathrm{s}}<t \leqslant(k+1) T_{\mathrm{s}}\end{cases}
$$

where $f_{\mathrm{s}}$ - switching frequency;

$T_{\mathrm{s}}-$ switching period, $T_{\mathrm{s}}=1 / f_{\mathrm{s}}$;

$\alpha_{k}$ duty cycle of the $k^{\text {th }}$ period;

Fourier series of Equation 6 is:

$$
\left\{\begin{array}{l}
V(t)=V_{\mathrm{s}}\left(2 \alpha_{k}-1\right)+\sum_{n=1}^{\infty} \frac{4 V_{\mathrm{s}}}{n \pi}\left|\sin \left(n \pi \alpha_{k}\right)\right| \cos \left(n \omega_{\mathrm{s}} t+\varphi_{n}\right) \\
\varphi_{n}=\arctan \frac{b_{n}}{a_{n}}=n \pi \alpha_{k}
\end{array}\right.
$$

For pure inductance load, the current signal can be expressed as:

$$
\begin{aligned}
I(t) & =\frac{V(t)}{\mathrm{j} \omega_{\mathrm{s}} L} \\
& =\frac{V_{\mathrm{s}}\left(2 \alpha_{k}-1\right)}{\mathrm{j} \omega_{\mathrm{s}} L}+\sum_{n=1}^{\infty} \frac{4 V_{\mathrm{s}}}{\mathrm{j} n \pi \omega_{\mathrm{s}} L}\left|\sin \left(n \pi \alpha_{k}\right)\right| \cos \left(n \omega_{\mathrm{s}} t+\varphi_{n}\right)
\end{aligned}
$$

The infinite series in Equation 8 causes the current ripple, and its amplitude decreases as the order increases. To improve signal-to-noise ratio, only the fundamental frequency $i_{1}(t)$ of coil current is extracted, namely, $n=1$. Where $i_{1}(t)$ is :

$$
\left\{\begin{array}{l}
i_{1}(t)=A_{1} \cos \left(\omega_{\mathrm{s}} t+\varphi_{1}\right) \\
\varphi_{1}=\arctan \frac{b_{1}}{a_{1}} \\
A_{1}=\frac{4 V_{\mathrm{s}}}{\mathrm{j} \pi \omega_{\mathrm{s}} L} \sin \left(\pi \alpha_{k}\right)
\end{array}\right.
$$

From the view of frequency domain, $A_{1}$ can be seen as amplitude of fundamental frequency of the current ripple. In the experiment, a series of signal processing stage including high-pass filter, abs circuit, low-pass filter etc. was adopted to extract $A_{1}$. And the duty cycle $\alpha_{k}$ is captured by the eCAP module of DSP28335. From Equation 5-9, the current ripple amplitude $A_{1}$ can be obtained as:

$$
A_{1}=\frac{4 V_{\mathrm{s}}\left(2 x+l_{\mathrm{c}} / \mu_{\mathrm{r}}\right)}{\mathrm{j} \pi \omega_{\mathrm{s}} \mu_{0} N^{2} A} \sin \left(\pi \alpha_{k}\right)
$$

So the air gap $\mathrm{x}$ can be written as:

$$
x=\frac{1}{2}\left[\frac{\pi \omega_{\mathrm{s}} \mu_{0} N^{2} A A_{1}}{4 V_{\mathrm{s}} \sin \left(\pi \alpha_{k}\right)}-\frac{l_{\mathrm{c}}}{\mu_{\mathrm{r}}}\right]
$$


When the ferromagnetic material is not saturated, air gap can be directly calculated from measured $A_{1}$ and $\alpha_{k}$ based on Equation 11. However, when saturated, $l_{\mathrm{c}} / \mu_{\mathrm{r}}$ increased rapidly, the calculated air gap based on Equation 11 cannot be used as system feedback.

\section{Influence of magnetic saturation on position estimation}

Electromagnetic force $F$ produced by a pair of poles is:

$F=\frac{B^{2} A \cos (\theta / 2)}{\mu_{0}}$

Where $\theta —$ the angle between the pole pair.

$B \_$magnetic flux density in the air gap

$B=\frac{N I \mu_{0}}{2 x+l_{\mathrm{c}} / \mu_{\mathrm{r}}}$

From Equation 12-13, it is obvious that the electromagnetic force $F$ is limited to magnetic flux density $B$. When AMB system suffers from external force disturbance, the controller will produce a larger current to reject the force disturbance, which will lead to magnetic flux saturation. When saturated, the coil equivalent inductance is not a monotonic function of the air gap. As a result, it makes no sense to calculate the coil inductance.

Ansoft-Maxwell is used for AMB modeling; different air gap with different coil current injection is carried out. The calculated coil equivalent inductance and air gap estimation is shown in Figure 3 and Figure 4.

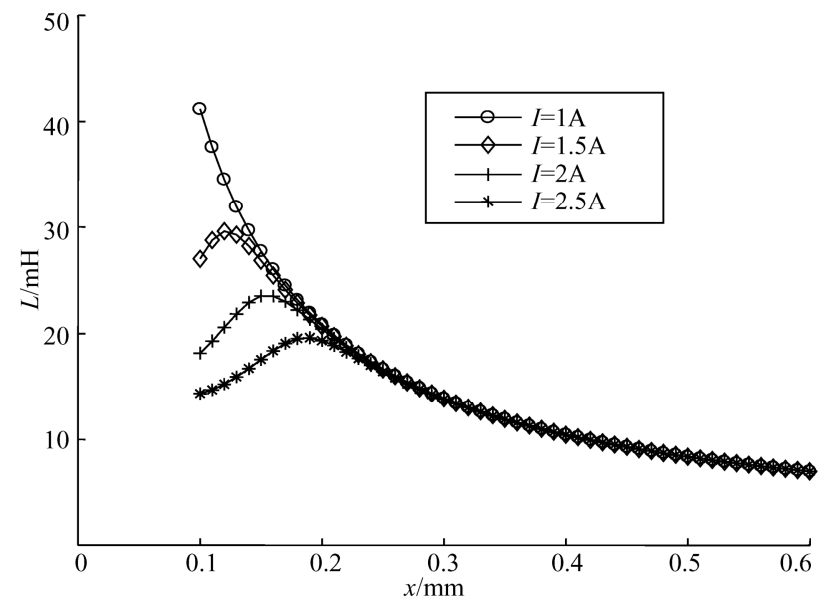

Figure 3 Variation of equivalent inductance with air gap under different coil currents

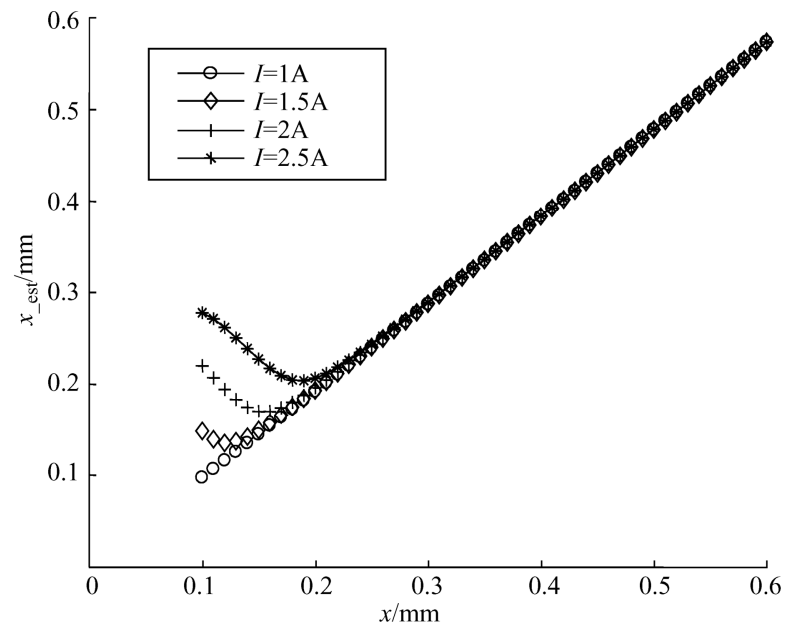

Figure 4 Position estimation under different coil currents 
From the figure above, it can be concluded that when unsaturated, the coil inductance only depends on air gap, no correlation with the coil current; when saturated, especially deep saturated, the measured inductance cannot be used, two air gap may be obtained based on this measurement which will lead to system unsteady.

\section{Cooperative rotor position estimation strategy}

\section{Strategy structure}

Figure 5 is the position estimation strategy for self-sensing AMB system using opposite pole pair. The controller uses position estimation $x_{\text {est }}$ as feedback, its output is the control current $i_{\mathrm{c}} \cdot i_{\mathrm{b}}$ is bias current. The upper and lower coil current is given by $i_{1}=i_{\mathrm{b}}+i_{\mathrm{c}}, i_{2}=i_{\mathrm{b}} \quad i_{\mathrm{c}}$ respectively.

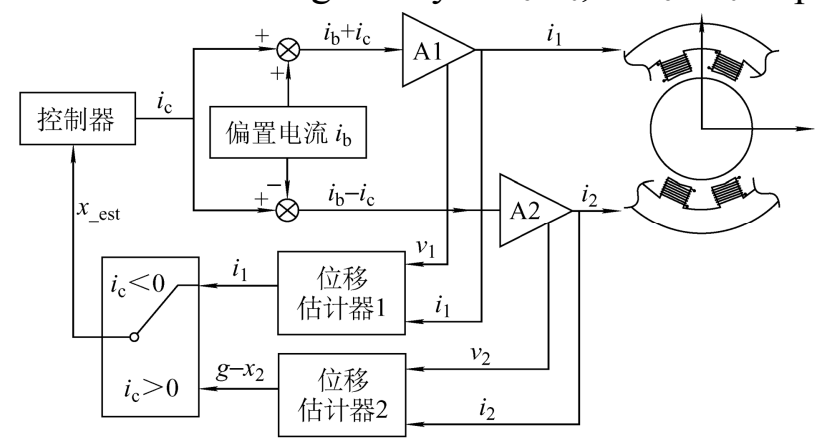

Figure 5 Position estimation strategy using opposite pole pair

The voltage and current of each pole were demodulated by the same position estimator to achieve the air gap respectively. When both pole are unsaturated: $x_{1}+x_{2}=g$ where $g$ is the total length of the air gap. The switch module is designed to select the unsaturated coil for estimation. If $i_{\mathrm{c}}<0, x_{1}$ is selected; If $i_{\mathrm{c}}>0, x_{2}$ is selected, $g \quad x_{2}$ acts as the feedback. The estimation result from the coil that has a smaller current is selected as position feedback to the control system. When $i_{\mathrm{b}}$ is reasonably designed, this strategy can avoid the influence of saturation on position estimation.

\section{Discussion on maximum $i_{b}$ for self-sensing AMB}

The bias current is chosen to produce bias flux. Due to the nonlinearity character of AMB, necessary bias flux should be given to set a working point for the purpose to decrease its nonlinearity. And a certain bias flux can also increase system stiffness and improve system stability.

However, for self-sensing AMB, magnetic saturation should be considered in the selection of bias current. On the premise of differential control, some extreme conditions for example, pulse or step external force disturbance, must be considered to make sure that there always is a pole that not saturated, or the position estimation strategy will be invalidation.

Obviously from Equation 13 that, small air gap and larger coil current lead to magnetic saturation. So the switch module selects the smaller current coil to avoid saturation. But the saturation still has the probability to appear in the smaller current coil when its air gap is too tiny. In the experiment platform, the air gap is limited to $0.1 \sim 0.6 \mathrm{~mm}$ by protection bearing. So that, if saturation does not happen when the air gap is $0.1 \mathrm{~mm}$, there will be no saturation in any other condition. This is the selection criterion for the max bias current $i_{\mathrm{b}}$.

\section{Experimental determination method of the maximum bias current}

Driven by current mode power amplifier, the coil current ripple is proportional to the air gap. When the air gap kept constant, the amplitude of current ripple remains constant if no saturated. Based on this characteristic, the maximum value of bias current $i_{\text {bmax }}$ could be obtained by experimental method as follows: inject current into a certain coil to produce electromagnetic force large enough to force the rotor firmly fixed on the protection bearing. In this case, air gap reach is minimum value $x_{\min }$. As mentioned above, the current ripple amplitude can remain constant until the ferromagnetic material reach saturation area. Increase the coil current and monitor the current ripple amplitude by oscilloscope until the amplitude begins to increase, and the current injected into the coil now is the maximum bias current $i_{\text {bmax }}$. On this platform, $i_{\text {bmax }}=1.2 \mathrm{~A}$. 


\section{Experiment}

\section{4-DOF AMB experimental platform}

Experimental platform include: current mode power amplifier, TMS320F28335 control center, DL1620 oscilloscope, A02-7112 asynchronous motor, 4 degree of freedom AMB, mallet, personal computer. The main parameters are shown in

\begin{tabular}{cc} 
Table. 1 Main parameters of 4-DOF experimental platfor & value \\
\hline Parameters / unit & 3.5 \\
Weight of rotor $/ \mathrm{kg}$ & 283 \\
Length of rotor $/ \mathrm{mm}$ & 207 \\
Center distance of AMB $/ \mathrm{mm}$ & 1.2 \\
Coil resistance $/ \Omega$ & 120 \\
Coil turns $N$ & 4.82 \\
Pole area $/ \mathrm{cm}^{2}$ & 50 \\
Bus voltage of power amplifier $/ \mathrm{V}$ & 3000 \\
Rated speed of the motor $/(\mathrm{r} / \mathrm{min})$ & 0.35 \\
Rated air gap $/ \mathrm{mm}$ & 20 \\
Inner diameter of protect bearing $/ \mathrm{mm}$ & 19.5 \\
Outer diameter of protect bearing $/ \mathrm{mm}$ &
\end{tabular}

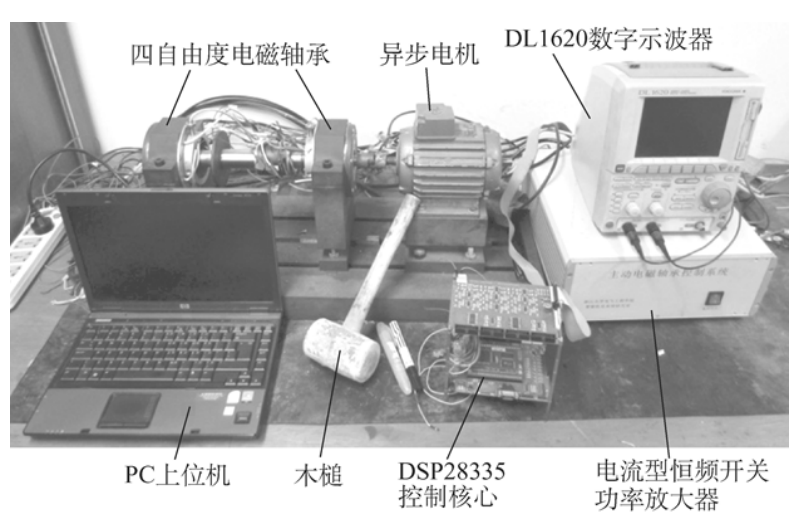

Figure 6 4-DOF experimental platform of AMBs

\section{Single freedom experiment}

Start the system without the motor, and make the rotor achieve static levitation. Knock on the rotor using mallet to simulate external force disturbance. The current in the upper coil will inevitably increase, and the finally reach saturation.

The coil current of left AMB is shown in Figure 7. Current in the upper coil rises rapidly after the knock and the current in the lower coil is limited to the minimum self-sensing current (set to $0.5 \mathrm{~A}$ in this experiment). Figure 8 shows the estimated air gap by upper and lower coil.

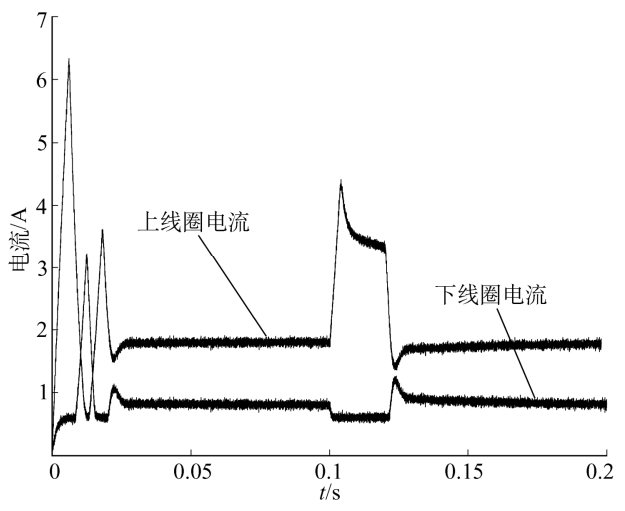

Figure 7 Current waveforms in the opposing coils of left AMB after being tapped by the mallet 


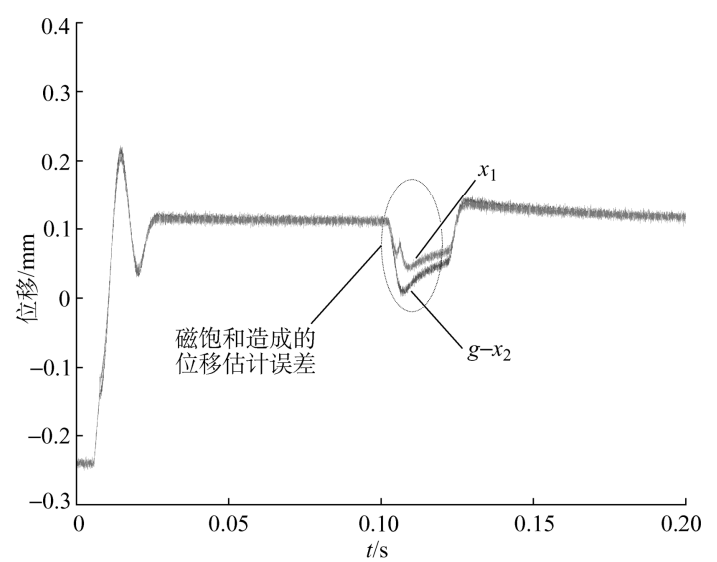

Figure 8 Estimator output of the opposing coils

The coordinate origin is set on the geometric center of the stator. Limited to the protect bearing, the range of the rotor is [ $0.25 \mathrm{~mm}, 0.25 \mathrm{~mm}]$ and the range of air gap is $[0.1 \mathrm{~mm}, 0.6 \mathrm{~mm}]$. Figure 8 showed that position estimation from both coil were nearly the same except the time rotor knocked, and this is caused by saturation. In this certain condition, the lower coil is selected as feedback to avoid the estimation error caused by saturation, and AMB system remains steady.

\section{Rotating experiment}

To verify the effectiveness and superiority, rotating experiment under different speed was carried out. In the experiment, for comparison, the right AMB adopts this strategy while the left one does not adopt. Figure 9 shows the rotor trajectory of each AMB (Unit: mm).

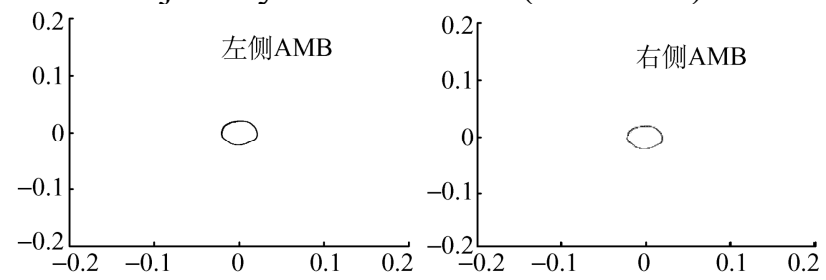

(a) $1000 \mathrm{r} / \mathrm{min}$

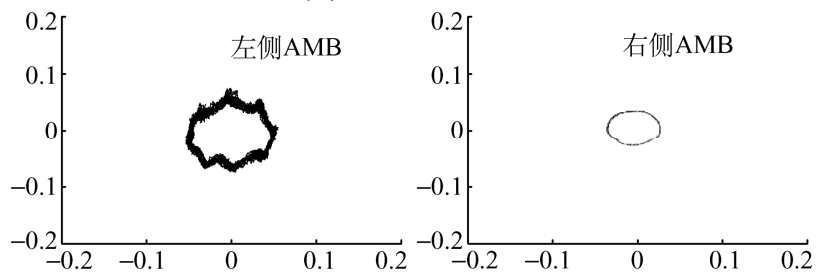

(b) $2000 \mathrm{r} / \mathrm{min}$

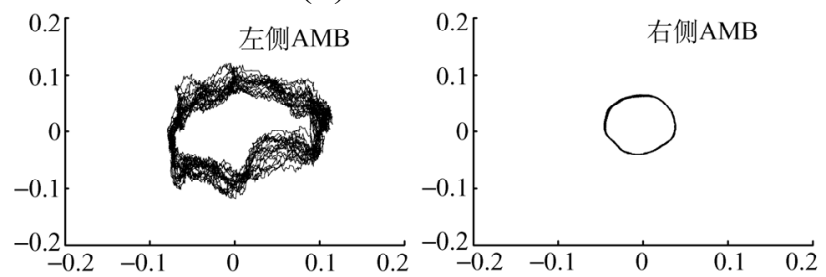

(c) $3000 \mathrm{r} / \mathrm{min}$

Figure 9 Rotor trajectory in both AMBs under different speeds

Due to mass unbalance, vibration amplitude of the rotor increases with its speed. To remain steady, the control current increases with the speed as a response. So, saturation always occurs at high speed. As shown in Figure 9, there is no obvious difference between the left and right AMB when $1000 \mathrm{r} / \mathrm{min}$. The left AMB seems unorganized at $2000 \mathrm{r} / \mathrm{min}$, but the system still remained steady. When the rotor speed reaches $3000 \mathrm{r} / \mathrm{min}$, the left AMB is nearly unsteady while the right AMB still has a satisfactory performance.

Then, configure both AMB with the cooperative rotor position estimation strategy. Figure 10 shows the trajectory of the rotor during the acceleration from $1500 \mathrm{r} / \mathrm{min}$ to $3000 \mathrm{r} / \mathrm{min}$. The trajec- 
tory seem a little unorganized during the acceleration, this is because the rotor is interference by the tangential force produced by the asynchronous motor.

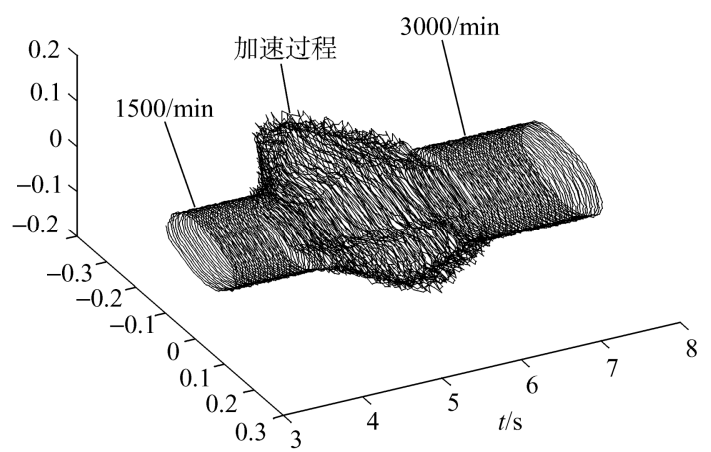

Figure 10 Rotor acceleration trajectory from $1500 \mathrm{r} / \mathrm{min}$ to $3000 \mathrm{r} / \mathrm{min}$

The rotating experiment proved that, the cooperative rotor position estimation strategy using opposite pole can effectively avoid magnetic saturation which would induce estimation error. This strategy can significantly improve the stability and robustness of self-sensing AMB system.

\section{Conclusions}

Inductor model of the AMB is established to discuss the influence from magnetic saturation to position estimation and the stability of whole AMB system. There must be an unsaturated pole in the opposite pole pair if the bias flux is chosen to an appropriate value. So, based on this theory, a strategy using the opposite pole pair is proposed to improve the robustness of self-sensing AMB system. Experiment verified the effectiveness of this strategy, the self-sensing AMB system can steadily operates at the speed of $0 \sim 3000 \mathrm{r} / \mathrm{min}$.

\section{References}

[1] Bleuler H. 1992. Survey of magnetic levitation and magnetic bearing types. JSME International Journal, Series 3: Vibration, Control Engineering, Engineering for Industry 35(3): 335-342.

[2] Vischer D. 1988. Sensorlose und spannugsgesteuerte magnetlager. Swiss Federal Institution Technology: Zurich Switzerland.

[3] Maslen E H.2006. Self-sensing for active magnetic bearings: overview and status. Proceedings of the 10th Internation Symposium on Magnetic Bearings: 10-15.

[4] Vischer D, 1990. Bleuler H. A new approach to sensorless and voltage controlled AMBs based on network theory concepts. Proceedings of the 2nd Internation Symposium on Magnetic Bearings: 301-306.

[5] Sivadasan K K. 1996. Analysis of self-sensing active magnetic bearings working on inductance measurement principle. IEEE Transactions on Magnetics, 32(2): 329-334.

[6] Maslen E H, Iwasaki T, Mahmoodian R. 2006. Formal parameter estimation for self-sensing. Proceedings of the 10th International Symposium on Magnetic Bearings: 529-536.

[7] Hanson B, Levesley M. 2004. Self-sensing applications for electromagnetic actuators. Sensors and Actuators, A: Physical, 116(2): 345-351.

[8] Hanson B M, Brown M D, Fisher J. 2001. Self sensing: closed-loop estimation for a linear electromagnetic actuator. Proceedings of the American Control Conference: 1650-1655.

[9] Garcia P, Guerrero J M, EI-Sayed I, et al. 2010. Carrier signal injection alternatives for sensorless control of active magnetic bearings. 1st Symposium on Sensorless Control for Electrical Drives: $78-85$.

[10]Mizuno T, Hirasawa Y. 2002. Self-sensing magnetic suspen- sion using an H-bridge type hysteresis amplifier operating in two quadrants. IECON Proceedings on Industrial Electronics Conference: $1818-1823$. 
[11] Schammass A, Herzog R, Buhler P, et al. 2005. New results for self-sensing active magnetic bearings using modulation approach. IEEE Transactions on Control Systems Technology 13(4): 509-516.

[12]Noh D. 1997. Self-sensing magnetic bearings driven by a switching power amplifier. Virginia USA: University of Virginia.

[13]Montie D T. 2003. Performance limitations and self-sensing magnetic bearings Virginia USA: University of Virginia. 